\title{
Joint embedding VQA model based on dynamic word vector
}

\author{
Zhiyang Ma ${ }^{1}$, Wenfeng Zheng ${ }^{\text {Corresp., } 1}$, Xiaobing Chen ${ }^{1}$, Lirong Yin ${ }^{2}$ \\ ${ }^{1}$ School of Automation, University of Electronic Science and Technology of China, Chengdu, P.R.China \\ 2 Department of Geography and Anthropology, Louisiana State University, LA, United States \\ Corresponding Author: Wenfeng Zheng \\ Email address: wenfeng.zheng.cn@gmail.com
}

The existing joint embedding Visual Question Answering models use different combinations of image characterization, text characterization and feature fusion method, but all the existing models use static word vectors for text characterization. However, in the real language environment, the same word may represent different meanings in different contexts, and may also be used as different grammatical components. These differences cannot be effectively expressed by static word vectors, so there may be semantic and grammatical deviations. In order to solve this problem[our paper constructs a joint embedding model based on dynamic word vector -- none KB-Specific network ((NKBSN) model which is different from commonly used VQA models based on static word vectors. N-KBSN model consists of three main parts: question text and image feature extraction module, self attention and guided attention module, feature fusion and classifier module. Among them, the key parts of N-KBSN model are: image characterization based on Faster R-CNN, text characterization based on Elmo and feature enhancement based on multi-head attention mechanism. The experimental results show that the N-KBSN constructed in our experiment is better than the other 2017 - winner( glove) model and 2019 - winner (glove) model .The introduction of dynamic word vector improves the accuracy of the overall results. 


\title{
1 Joint embedding VQA model based on dynamic word vector
}

2

3

Zhiyang $\mathrm{Ma}^{1}$, Wenfeng Zheng ${ }^{2}$, Xiaobing Chen ${ }^{3}$, Lirong Yin ${ }^{4}$

${ }^{1}$ University of Electronic Science and Technology of China, Chengdu, 610054, P.R.China

${ }^{2}$ University of Electronic Science and Technology of China, Chengdu, 610054, P.R.China

${ }^{3}$ University of Electronic Science and Technology of China, Chengdu, 610054, P.R.China

${ }^{4}$ Department of Geography and Anthropology, Louisiana State University, Baton Rouge, LA 70803, USA

\section{Corresponding Author:}

Wenfeng Zheng ${ }^{2}$

Xiyuan street 2006, Chengdu 610054, Sichuan, P. R. China

Email address:wenfeng.zheng.cn@gmail.com

\begin{abstract}
The existing joint embedding Visual Question Answering models use different combinations of image characterization, text characterization and feature fusion method. But all the existing Visual Question Answering models use static word vectors for text characterization. However, in the real language environment, the same word may represent different meanings in different contexts, and may also be used as different grammatical components. These differences cannot be effectively expressed by static word vectors, so there may be semantic and grammatical deviations. In order to solve this problem, our paper constructs a joint embedding model based on dynamic word vector -- none KB-Specific network ((N-KBSN) model which is different from commonly used VQA models based on static word vectors. N-KBSN model consists of three main parts: question text and image feature extraction module, self attention and guided attention module, feature fusion and classifier module. Among them, the key parts of N-KBSN model are: image characterization based on Faster R-CNN, text characterization based on ELMo and feature enhancement based on multi-head attention mechanism. The experimental results show that the N-KBSN constructed in our experiment is better than the other 2017 — winner (glove) model and 2019 - winner (glove) model .The introduction of dynamic word vector improves the accuracy of the overall results.
\end{abstract}

Keywords: Faster R-CNN， ELMo， MA，VQA 


\section{Introduction}

VQA(Visual Question Answering) system takes a picture, and a free and open natural language question about this picture as input, and generates a natural language answer as output. VQA has many potential applications. The VQA system can be integrated with other research areas, such as social media , e-commerce, medical Science(Tang et al. 2020),seismology(Yin et al. 2019; Zheng et al. 2016), disabilities(Gelšvartas et al. 2016), gaming(Atkinson et al. 2019) and chatbots(Klopfenstein et al. 2018). Since the appearance of visual question answering task in 2015, a large number of VQA model belong to the joint embedding model. Firstly, visual information and question text information are respectively characterized in the joint embedding model, and then image features and text features are fused and finally predict the answer through the classifier.Because the model of this architecture is easy to be trained, researchers have tried a lot of different image feature extraction methods, different text feature extraction methods, and different fusion methods of the two modes.

In the aspect of text characterization, Zhou et al proposed the iBOWIMG model(Zhou et al. 2015), and transferred the pre-trained GoogleNet(Szegedy et al. 2015) to extract image features. Gao et al thought that the question and answer are different in syntactic structure, so they used two independent LSTM networks to encode the question and decode the answer, and combined the convolutional neural network to form the mQA model(Gao et al. 2015). Lin et al proposed a dual CNN model. They applied the convolutional neural network CNN to both the encoding of the image and the feature extraction of the question text, and used a multimodal convolution layer to output the joint eigenvectors(Ma et al. 2015).

In the aspect of image feature extraction, in addition to different models using different pre-trained CNN, Noh et al thought that the deep convolution neural network with single weight configuration can not effectively deal with different problems(Noh et al. 2016). They added a dynamic parameter layer to the convolutional neural network CNN.

In addition to using different methods to extract image and text features, cross mode feature fusion method has been widely studied. Malinowski et al proved that the accuracy of the system is related to the feature vector fusion method by comparing different feature vector fusion methods(Malinowski et al. 2015). Antol et al used the element by element multiplication feature fusion method(Antol et al. 2015). Fukui et al thought that in the external multiplication operation between vectors, the interaction between all elements was more active and can retain more abundant feature information, so a more complex Modal compact bilinear pooling method (MCB) was proposed(Fukui et al. 2016). Saito et al. thought that different feature fusion methods would retain different levels of features. Therefore, the model Dualnet(Saito et al. 2017) which combines element by element addition and element by element multiplication is proposed. 
Attention mechanism has been proved to be effective in a large number of deep learning tasks, attention mechanism is also widely used in VQA model. Chen et al. firstly introduced attention mechanism into visual question answering task, and proposed a configurable convolutional neural network (ABC-CNN) based on attention mechanism to reduce the influence of irrelevant regions(Chen et al. 2015).The test results on Toronto COCO-QA(Ren et al. 2015a), DAQUAR(Malinowski \& Fritz 2014), and VQA(Antol et al. 2015) all achieved optimal results, which proved the effectiveness of attention mechanism in improving VQA tasks.

This paper analyzes the following reasons for the excellent performance of joint embedding model in VQA challenge:

1. Attention mechanism is introduced. In 2016, the winning model(Ilievski et al. 2016) proposed the dynamic attention (FDA) model. The purpose is to dynamically allocate weights to different regions of the image according to the keywords in the question, so as to obtain the combination of global features and local features of the image. The top-down and bottom-up image attention mechanisms of paper(Anderson et al. 2018) were used in the winning models in 2017 and 2018, and the multi-head attention mechanism of Transformer(Vaswani et al. 2017) was used in the winning model in 2019. The introduction of attention mechanism can reduce the interference of irrelevant features, improve the computational efficiency, and improve the interpretability to a certain extent

2. The limitations of VQA2.0 dataset. VQA challenge takes VQA2.0 as the data set. However only $5.5 \%$ of all questions require common knowledge or external knowledge in VQA2.0, which means that no additional information is needed to answer most of the questions(Wang et al. 2015b)Error! Reference source not found.. However, in the open questions in reality, questions involving common sense or external knowledge exist widely. Therefore, VQA2.0 dataset has limitations, which makes the model only need to focus on images and text, so joint embedding model has become the main architecture.

3. Thanks to the progress of image recognition and natural language processing model. The joint embedding model has flexible combination pattern, and it is easy to transfer the model which performs well in other tasks to the forming of a new model.

As shown in the table 1, the existing joint embedding models use different combinations of image characterization, text characterization and feature fusion methods, but all existing models use static word vectors for text characterization. The static word vector uses a corpus as the data set to train and get the distributed representation of each word. The advantage of this representation method is that the word vectors are obtained through pre-training. Therefore, when it is applied to different downstream tasks, there is no need to retrain and the computational efficiency is improved. However, in the real language environment, the same word may represent different meanings in different contexts, and may also be used as different grammatical components. These differences 
104

105

106

107

108

109

110

111

112

113

114

115

116

117

118

119

120

121

122

123

124

125

126

127

128

129

130

131

132

133

134

135

136

137

cannot be effectively expressed by static word vectors, so there may be semantic and grammatical deviations.

In order to solve the problem of static word vector, our experiment constructs a joint embedding model based on dynamic word vector -- none KB specific network (N-KBSN) model. Our paper will focus on the N-KBSN model, and use VQA2.0 dataset for training. N-KBSN consists of three main parts: question text and image feature extraction module, self attention and guided attention module, feature fusion and classifier. Faster R-CNN, which is excellent in multi-label detection, is used for image feature extraction.According to M. E. Peters et al (Peters et al. 2018), the ELMO representations ideall model both (1) complex characteristics of word use (e.g., syntax and semantics), and (2) how these uses vary across linguistic contexts (i.e., to model polysemy). And ELMO representations can be easily added to existing models and significantly improve the state of challenging NLP problems. Inspired by this, in our VQA experiment, ELMo model which can obtain context information is used for feature extraction of question text, and multi-head attention mechanism(Vaswani et al. 2017) learned from transformer is used to realize image self attention (V-SA), question text self attention (Q-SA) and image attention guided by the question (Guided Attention, GA), and the answer is predicted by feature fusion. The infrastructure of N-KBSN model is shown in Fig 1.

\section{2、DATA}

Since 2014, several high-quality VQA data sets have been proposed: DAQUAR(Malinowski \& Fritz 2014)、 COCO-QA(Ren et al. 2015a)、VQA(Antol et al. 2015)、VQA 2.0(Goyal et al. 2017)、CLEVR(Johnson et al. 2017)、KB-VQA(Wang et al. 2015a)、FVQA(Wang et al. 2017).Among which, the KB-VQA and FVQA are knowledge-based dataset.

1. DAQUAR dataset contains 1449 images, most of which are indoor scenes, which greatly limits the richness of the scene of the dataset, which is a major disadvantage of the dataset. The data set consists of training set and test set. The question and answer pairs are generated by algorithms or provided by human volunteers and are generated according to the given template.

2. COCO-QA contains 123287 real scene images from MS COCO. The questions are divided into four types: object recognition, color recognition, counting and location query. In the actual test process, DAQUAR data set has been found to be able to obtain high accuracy only by simple guessing the answer, which makes the high accuracy rate appear a great deviation, which cannot fairly test the reasoning ability of the system. In order to overcome this shortcoming, COCO-QA eliminates some answers with extremely low and extremely high frequency, making the frequency of common answers decrease from $24.98 \%$ to $7.30 \%$. 
3. VQA data set is an important turning point in the development of visual question answering field. Before that, the question types of previous data sets were limited to some templates, which made the data sets unable to test the performance of visual question answering system in real context. For example, DAQUAR limited the answers to 16 basic colors and 894 object categories. The questions and answers in VQA dataset are unlimited and open, and all of them are generated by human beings. At the same time, the number of pictures is two orders of magnitude higher than DAQUAR, reaching 254731, which greatly improves the capacity of the data set. The VQA dataset contains not only the 204721 images of real scenes and 50000 synthetic abstract scene images are provided, which enriches the diversity of database scenes and facilitates high-order scene reasoning and complex spatial reasoning.

4. VQA 2.0. Aimed at the question of language bias in VQA dataset, VQA 2.0 enhances the visual information of VQA dataset by adding new Confused data to the original VQA dataset. Confused data is organized in the form of (image I, question Q, answer A) just like the original data. The difference is that the newly added image is similar to the original image, but the answer to the same question $\mathrm{Q}$ gives a different answer A. For the same question, facing different pictures, we need to get different answers, which requires that the system not only can understand the natural language questions, but also can accurately identify the differences between pictures. This balanced method can filter out the algorithms that weaken image understanding, and enhance the importance of image understanding in VQA tasks. The supplementary VQA 2.0 contains 1.1 million pairs of image -question and 200000 real scene images with 1300 million questions. The data volume is almost twice as much as that of VQA data set, and has become a new test standard for open questions.

5. CLEVR. In order to measure the reasoning ability of visual question answering system more accurately, Johnson et al (Johnson et al. 2017) proposed a diagnostic CLEVR data set which combines language and basic visual reasoning.

In this experiment, it is enough to use VQA2.0 data set to train visual question answering model. The specific information about the data set is introduced in section 3.4.1.

\section{3、Method and Experiments}

\subsection{Image characterization based on FasterR-CNN}

Target detection aims at locating the target accurately and quickly from the image, and recognizing the characteristics of the object such as its category. Traditional target detection algorithms select regions, extract features and classify them by sliding window, such as deformable components Model (DPM) method(Felzenszwalb et al. 2009). Most of these traditional target detection algorithms have poor performance and high time complexity. Deep learning has been performing 
172 well in VQA tasks, and there are a large number of excellent target detection algorithms, such as 173 R-CNN(Girshick et al. 2014), SPP-Net(He et al. 2015), Fast R-CNN(Girshick 2015), Faster R174 CNN(Ren et al. 2015b), Mask R-CNN (He et al. 2017), YOLO(Redmon et al. 2016) and so on. 175 Because Faster R-CNN has shown excellent performance in various target recognition tasks, our 176 experiment uses Faster R-CNN to extract image features.

177 FasterR-CNN is divided into four modules: convolution layer, regional candidate network (RPN), 178 ROI pooling and classifier (Ren et al. 2015b). CNN and its variants are used by the convolution layer to extract image features. The RPN network is used to predict the candidate regions. The network slides on the image features output by CNN. The network predicts the category score In each spatial region. The feature map and candidate region of the image are combined with the pooling layer of interested region to get the region feature map. Then, the fully-connected layer and classifier are used to predict the category of each region, and the detection frame of the object is obtained by candidate frame regression.

The N-KBSN model uses ResNet-101 which has been pr-trained on Imagenet(Russakovsky et al. 2015) and Faster R-CNN trained on Visual Genome(Krishna et al. 2017) to extract image features. Given the image $\mathrm{I}$, the model extracts m non-fixed size image features $X=\left\{x_{1}, x_{2}, \ldots, x_{m}\right\} x_{\mathrm{i}} \in R^{D}$, and each image feature encodes an image region. The feature dimension of each image region is 2048. For the feature map output by the convolution layer, the model uses non-maximum suppression and IOU thresholds to select the top candidate regions. By setting a threshold of target detection probability, the network obtains a dynamic number of detected objects $m \in[10,100]$, and uses zero padding to make $\mathrm{m}=100$. For each selected region $\mathrm{I}, x_{i}$ is defined as the mean pooling result of the feature graph of the region, and $x_{i}$ of $\mathrm{m}$ regions are spliced into the final image feature. Therefore, each input image will be transformed into a $100 \times 2048$ image feature for subsequent attention module.

\subsection{Text characterization based on ELMo}

As shown in table 1, text characterization of previous visual question answering models is to obtain static word vectors through corpus learning, each word corresponds to a certain real number vector. This fixed vector does not perform well in dealing with the polysemy of words. Both Chinese and English words have the phenomenon of polysemy, the meaning of the same word changes in different contexts. In order to solve the problem of polysemy, dynamic word vector is proposed. And ELMo and BERT are the representatives. ELMo improves the accuracy of the model in multiple NLP tasks. Therefore, our experiment introduces ELMo model to deal with text in VQA tasks, and combines attention mechanism which is similar to Bert in subsequent 
205

206

207

208

209

210

211

212

213

214

215

216

217

218

219

220

221

222

223

224

225

226

227

228

229

230

231

232

233

234

processing.

ELMo(Peters et al. 2018) uses two stages to obtain the word vector. The first stage is to train a deep bi-directional language model (biLSTM) with a large number of text corpus; the second stage is to extract the internal state of each layer of the network corresponding to the word from the pretrained network, and transforms it into the word vector through functions. The structure of ELMo model has been shown in Fig 1.

Firstly, the maximum length of the sentence is cut to 14, and the sentences with less than 14 words are supplemented to 14 by zero filling. Each word is transformed into an initial word vector of 50 dimensions, that is, the "context free" initial word vector $y_{k}^{L M} \in \mathrm{i}^{50}$, assuming that the number of layers of the bi-directional language model is $\mathrm{L}=2$, the number of hidden layer nodes is $H_{\text {dim }}$, and the output dimension is output $t_{\operatorname{dim}} \in R^{d}$, then ELMo is $E M \mathcal{O}^{\text {ask }} \in \mathrm{i}^{2 d}$, the output text feature $Y \in \mathrm{i}^{n \times 2 d}$.

\subsection{Multi-head attention mechanism}

As mentioned in the introduction, the introduction of attention mechanism helps the neural network to improve the prediction accuracy and reduce the computational complexity. VQA tasks need to process multi-modal data such as images and texts, which requires more efficient computation than tasks that only need to process single-mode data. At the same time, the input image and question text have a high correlation, so the interaction betwen the data of the two modals also has a significant impact on the accuracy of the results. For the above two requirements, N-KBSN uses the multi-head attention (MA) mechanism of Transformer(Vaswani et al. 2017) to realize the self attention of image (V-SA), question text self attention (Q-SA) and image attention (GA) guided by questions(Yu et al. 2019).

The essence of attention mechanism is to find a way to assign appropriate weight to the existing information and improve the accuracy of the output. According to the multi-head attention (MA) mechanism of Transformer(Vaswani et al. 2017), Attention function can be described as mapping query to some key value pairs and obtaining the output. Suppose the query matrix $Q=\left\{q_{1}, q_{2, \ldots .} q_{m}\right\}$

, where the query vector $q_{i} \in R^{1 \times d_{q}}$; the key matrix $Q=\left\{k_{1}, k_{2, \ldots .} k_{n}\right\} k_{j} \in R^{1 \times d_{k}}$, value matrix $Q=\left\{v_{1}, v_{2, \ldots} v_{n}\right\}$, where the value vector $v_{i} \in \mathrm{i}^{1 \times d_{v}}$, then the attention feature can be obtained by weighting the value matrix, and the weight can be obtained by the query matrix and key matrix(Vaswani et al. 2017). 
235 In order to further improve the expression ability of attention features, multi-head attention 236 mechanism(Vaswani et al. 2017) is introduced. The realization of multi-head attention mechanism

237 is to input Q, K, V into h linear layers with different weights. Finally, $h$ attention features are 238 stitched together and the attention features of the expected dimension are obtained through a linear 239 layer.

240 Based on the idea of multi-head attention mechanism, this paper uses three kinds of attention 241 features: self attention of picture (V-SA), self attention of question text (Q-SA), and image 242 attention guided by question(GA). Assuming that the text word vector matrix is $Y$ and the image 243 feature map is $X$, then when calculating $\mathrm{V}-\mathrm{SA}, \mathrm{Q}=\mathrm{K}=\mathrm{V}=\mathrm{X}$, that is, the output image feature 244 is $\mathrm{SA}=\mathrm{MA}(\mathrm{X}, \mathrm{X}, \mathrm{X})$; when calculating $\mathrm{Q}-\mathrm{SA}, \mathrm{Q}=\mathrm{K}=\mathrm{V}=\mathrm{Y}$, that is, the output text feature is $245 \mathrm{SA}=\mathrm{MA}(\mathrm{Y}, \mathrm{Y}, \mathrm{Y})$; when calculating the guided attention feature, $\mathrm{Q}=\mathrm{Y}$ is the word vector 246 matrix, $\mathrm{K}=\mathrm{V}=\mathrm{X}$ is the image feature matrix, and the word vector and image feature vector have 247 the same dimension, that is, the output image feature guided by the question is GA = MA (Y, X, $248 \mathrm{X})$. A common attention module (MCA) is composed of three kinds of attention combinations( $\mathrm{Yu}$ 249 et al. 2019) which takes the the original image feature and text feature as input, and the output is 250 the image and text feature through attention mechanism.

251 In order to improve the use of deep attention mechanism to extract higher-level features, MCAN 252 paper(Yu et al. 2019) proposed Encoder-Decoder and Stacking which are two ways of cascading 253 MCA layer. Among them, stacking takes the output of the upper layer as the input of the next 254 layer, and Encoder-Decoder takes the question self attention feature of the last layer as the query 255 matrix of each layer.

256 According to the performance of the two cascading methods in multiple tasks, N-KBSN model 257 uses the cascade mode of Encoder-Decode.

258

259

260

261

262

263

264

265

266

267

268

\subsection{Experiment}

Our experiment builds a series of VQA models with different network structures or parameter settings, and train and evaluate the models using the general open question-answering data set VQA2.0(Goyal et al. 2017). The purpose of the experiment is to compare the influence of dynamic word vector and static word vector on the accuracy of results, and find the optimal N-KBSN model by a large number of experiments. The whole code is implemented in Python, with pytorch as the machine learning platform, and a computer training model with $32 \mathrm{G}$ memory and GPU is used.

\subsubsection{Experimental setup}

(1) Data set. In this experiment, VQA2.0 dataset was used to train the model. The data set is divided into three data subsets: train / val / test, which contains 80000 images +444000 question and answer pairs, 40000 images +214000 question and answer pairs, 80000 images 

+448000 question and answer pairs. The answers include Yes or No, quantity and others. The pictures are real scenes extracted from MS-COCO dataset. In addition, according to the images which are both in VQA2.0 and Visual Genome, 490000 question and answer pairs are extracted from Visual Genome to enhance the training set.

(2) Evaluation method. In order to train and test the open questions, the VQA2.0 dataset designs the questions manually. Each picture has three questions proposed by human beings. The answers are all open-ended, and the evaluation method of these open-ended answers also introduces artificial evaluation mechanism: for the same open question, ten people answer it separately. If three or more subjects provide the same answer, the answer is regarded as the correct answer. Therefore, the text uses accuracy as the evaluation parameter, including overall accuracy and sub-item accuracy. According to the type of answer, the accuracy of sub-items can be divided into Yes or No, count and others.

(3) Parameter setting of fixed module. The purpose of our experiemnt is to compare the effect of different word vector embedding methods on the accuracy of the model, so other parts of the model should keep the same parameter settings. Specifically, for the image feature extraction module, the number of candidate image regions of Fast $\mathrm{R}-\mathrm{CNN}$ is $\mathrm{m}=100$, and the feature dimension of a single image area is $x_{i}=2048$, so the single image feature $X \in \mathrm{i}^{100 \times 2048}$. The hidden layer dimension of MA attention in the SA and GA module is $d=512$, the number of heads $h=8$, that is, the hidden layer dimension of each head $_{h}=d / h=64$, and the number of MCA layers L $=6$. We select words or phrases with more than 8 occurrences from all the answers, and construct an answer dictionary with the size of $\mathrm{n}=3129$, that is, the number of classified categories is 3129 .

The activation function uses ReLU. The parameters of Adam optimizer are $\beta_{1}=0.9$, $\beta_{2}=0.98$ and the learning rate is $\min \left(2.5 t e^{-5}, 1 e^{-4}\right)$, where $t$ is the number of trained epochs. Starting from the 10th generation, the learning rate of every two generations decreases to $1 /$ 5 of the current. The number of batch samples is 32, and the training epoch is 13 .

With the rest of the fixed model unchanged, this experiment will construct models using different text characterization methods to evaluate the impact of dynamic and static word vectors on the accuracy of the results. And the technical details of our model is shown in Fig2.

\subsubsection{Model based on static word vector}

In the experiment, the representative and widely used pre-trained word $2 \mathrm{vec}$ and Glove word 
300

301

302

303

304

305

306

307

308

309

310

311

312

313

314

315

316

317

318

319

320

321

322

323

324

325

326

327

328

329

330

331

332

vectors are selected in the experiment, and a single layer LSTM network is cascaded to convert the feature dimension into 512 dimension, which is convenient for the fusion with image features subsequently. The word2vec word vector uses the word vector obtained from word2vec model which has been trained on Google News.And the Google News contains 100 billion words and phrases. Word2vec word vector is composed of three million words and phrases.

The Glove word vector is obtained by being trained on the corpus of Wikipedia and twitter, which contains 2 million 300 dimensional vectors. In order to use the static word vector, the question text of the input sample is cut into a 14 word sequence, and then the static vector of each word is obtained by using the look-up table. If there is no pre-traind word vector in the data set, the word vector is initialized as zero vector.

\subsubsection{Model based on dynamic word vector}

Different from the static vector configuration, in order to obtain the dynamic word vector, the pretrained deep bidirectional LSTM network (biLSTM) is embedded into the model, and the weight parameters and scale parameters of ELMo word vector are obtained by training. In order to further explore the best ELMo parameters, three pre-trained ELMo models with different parameters are selected in this experiment, which are $E M O_{s} /$ 且 $M O_{m} /$ E $M O_{1}$. The parameters, hidden layer size, output size, ELMo size and LSTM size are shown in table2. As shown in the table, the main differences of the three different ELMo models lie in model depth and the dimension of word vector. Theoretically, the deeper network depth, the greater capacity. And the higher dimensional word vector can contain more semantic information. In the same way, the question text is cut into the sequence containing 14 words, and the whole word sequence is taken as input. Through twolayer biLSTM network, ELMo word vector containing the context is obtained. Then the obtained ELMo word vector is unified into 512 dimensions through a LSTM network, and the fused feature $Z \in \mathrm{i}^{512}$

Statistics of several word vectors are shown in table 3. Because ELMo model uses character-level coding, even for words that do not exist in the corpus, the initial word vector can still be obtained, and then the ELMo word vector can be obtained. Therefore, the number of ELMo word vectors is theoretically infinite.

In order to facilitate the analysis of experimental results, the models with different text features are represented as follows baseline(w2v), baseline (glove), $N-K B S N(s), N-K B S N(m), N-$ $\operatorname{KBSN}(l)$.

All in all, in this experiment, VQA2.0 dataset is used to train and evaluate the six models: baseline(random), baseline (w2v), baseline (glove), $N-K B S N(s), N-K B S N(m) N-K B S N(l)$.Among 
333

334

335

336

337

338

339

340

341

342

343

344

345

346

347

which, the word vector of baseline(random) was randomly initialized and cascaded with LSTM network.

At the same time, the model comparison also includes the 2017-winner(glove) and 2019 winner(glove) model that has won the VQA challenge in the past years

\section{4、 Results of Finding}

\subsection{Results of VQA comparison experiment}

The experimental results of baseline(random), baseline (w2v), baseline (glove), $N-K B S N(s), N-$ $K B S N(m) N-K B S N(l)$ on the val set are shown in table4.

\subsection{Comparison about $\mathrm{N}$ - KBSN(m) model and baseline (glove) model}

As shown in table 5, the experimental results of baseline (glove) is better than baseline ( $w 2 v)$, and $N-K B S N$ model using ELMo dynamic vector are better than baseline(glove). Specifically, the ELMo model parameters of N - KBSN (1) are more than three times of that of N - KBSN (m), but the accuracy rate is not significantly improved. Consequently, we would conduct comparison experiments between $N-K B S N(m(m)$ and baseline (glove) to find out where the difference lie in. In order to quantitatively analyze the difference between $N-K B S N(m(\mathrm{~m})$ and baseline (glove), the VQA2.0 dataset is randomly sampled to form training subsets with the size of $10 \%, 30 \%, 50 \%$, $70 \%, 90 \%$ and $100 \%$ of the original size respectively, and the two models are trained according to the same parameter configuration in previous experiments. The overall accuracy of the two models on the val dataset is shown in table 5, and the diagram is shown in Fig 3.

In order to qualitatively analyze the difference between the results of $N-K B S N(m)$ and baseline (glove), we compare and analyze the prediction results of the two models in the val dataset, and uses Res(ELMo) and Res(glove) to represent their result sets respectively. The size of Res(ELMo) and Res(glove) is the number of questions in the val set: 214354 . The number of questions with different answers given by the two models is 52990, accounting for about $1 / 5$ of the total. Among the different answers given, Res(ELMo) answered correctly and Res(glove) answered wrong accounted for $27.8 \%$, Res(glove) answered correctly while Res (ELMo) answered wrong accounted for $26.4 \%$, the two both answered wrong accounted for $45.8 \%$, and the two both answered wrong accounted for $0 \%$, as shown in table 6 .

In order to further qualitatively analyze the possible reasons why $\mathrm{N}-\mathrm{KBSN}(\mathrm{m})$ is better than baseline (glove), we selected some samples from the answers, as shown in Fig 4. 
363

364

365

366

367

368

369

370

371

372

373

374

375

376

377

378

379

380

381

382

383

\subsection{Comparison between existing models}

As shown in table 5, $N-K B S N(l)$ performs best on the val set. The results of the model and 2017

— winner glove) and 2019 - winner (glove) on Test-dev and Test-std are shown in table 7.

\section{5、 Discussion}

\subsection{Discussion about VQA experiment results}

In the real language environment, the complex characteristics of word use (e.g., syntax and semantics), and how these uses vary across linguistic contexts cannot be effectively expressed by static word vectors, so there may be semantic and grammatical deviations. So we introduce dynamic word vector into the VQA models, and do experiments on the baseline(random), baseline (w2v), baseline (glove), $N-K B S N(s), N-K B S N(m), N-K B S N(l)$ models. As shown in the table 4 , in terms of the accuracy of the answer type, the experimental results of all models are as follows: $\mathrm{Y} / \mathrm{N}>$ others $>$ count, and the accuracy difference of any two remains stable in the results of different models. This shows that the accuracy difference of the single type is independent of the model and comes from the characteristics of the question itself and the data set. For example, the expected accuracy rate of the sample with the answer type of Yes or No is 50\%, while that of the answer type of count is very low. The difference of difficulty between the two types of answers leads to that the accuracy of the Yes or No type is always higher than that of the count type.

The first five models all use static word vectors as text features. baseline (random) uses randomly initialized text features, which contains less semantic and grammatical information than the pretrained word vector, so its accuracy is the lowest.2017 — winner(glove) has a lower accuracy than the baseline model because of its simpler attention mechanism. Comparing the results of baseline(w2v) and baseline(glove), we can see that even if the corpus of word2vec word vector is nearly 20 times as large as that of glove word vector, the model based on glove still takes the lead in all aspects, even if other parts are the same. As the paper(Pennington et al. 2014) said, the glove word vector uses co-occurrence matrix. Compared with word2vec word vector, which only uses local context information, it introduces the global information of corpus and improves the representation ability. Therefore, even if a larger corpus is used, the representation ability of word2 vec word vector is still worse than that of glove word vector.

The last three models are the N-KBSN model proposed in our experiment. From the results, we can see that compared with baseline(glove), the accuracy of each item is significantly improved, which proves that the dynamic word vector can improve the text feature representation ability of the model to a certain extent, and then improve the overall result accuracy. And the accuracy of the three N-KBSN models are all higher than the 2019 - winner(glove). 
396

397

398

399

400

401

402

403

404

405

406

407

408

409

410

411

412

413

414

415

416

417

418

419

420

421

422

423

424

425

426

427

428

Compare the ELMo model with three different parameters, it is not difficult to find that with the increase of model depth and feature dimension, the overall accuracy rate is improved, but the improvement range is gradually reduced. Specifically, the parameters of ELMo model of $N$ $K B S N(l)$ are more than three times of that of $N-K B S N(m)$, but the accuracy rate is not significantly improved.

\subsection{Discussion about the comparison of $\mathrm{N}-\mathrm{KBSN}(\mathrm{m})$ model and baseline (glove) model}

According to the above Fig3 and table 5, with the increase of training data, their accuracy rates monotonically increase, and the rising speed gradually decreases, making the accuracy rate tend to be stable. This shows that increasing the amount of training data can help improve the accuracy, but the improvement will tend to saturation. It is worth noting that only $10 \%$ of the training data can get better accuracy.

We want to determine whether the difference of accuracy is statistically significant between the $N$

- $K B S N(m)$ and baseline (glove). We use the Wilcoxon signed rank test and the results showed in Fig 5 indicate $\mathrm{p}<0.05$. Consequently, the difference is statistically significant.

The accuracy of $N-\operatorname{KBSN}(m)$ is always higher than baseline (glove), which shows that dynamic word vector can improve the accuracy of the model. With the increase of data, the difference of accuracy between the two models gradually increases. This is because the dynamic word vector can effectively represent the performance of polysemous words. Larger training data means that it is more likely to include the use of words in different contexts, while static word vector can not effectively deal with this situation.

We compare and analyze the prediction results of the $N-K B S N(m)$ and baseline (glove) in the val dataset as shown in table6. From the overall answer difference, the number of correct answers of $N-K B S N(m)$ is slightly higher than that of baseline (glove). As shown in Fig4, Res(ELMo) correctly identifies the color of bath curtain, while Res(glove) answers white. The possible reason is that the model mistakenly identifies the color of shower instead of shower curtain. Similarly, in Fig (b), Res(glove) wrongly counts the phrase people while Res(ELMo) correctly counts the phrase elderly people. Fig (c) shows that Res(ELMo) performs better in recognizing phrases in long sentences rather than just words.

\subsection{Discussion about comparison with existing models}

The result on the test set shown in table 7 indicates that the $N-K B S N(l)$ proposed in our experiment is better than the other 2017 - winner (glove) and 2019 - winner (glove)models in each index, and the results are consistent with the results on the val set, which proves that the improved text characterization method can improve the prediction accuracy. 
429 We compared the difference between $N-K B S N(l)$ and 2017 - winner ( glove), and the difference 430 between $N-K B S N(l)$ and 2019 - winner( glove). We want to determine whether the difference of 431 accuracy is statistically significant between the visual question answering model based on dynamic 432 word vector and the visual question answering model based on static word vector. We use 433 Wilcoxon signed rank test to test the statistical significance, and the statistical results are shown 434 in Fig6 and Fig7. According to the statistical results, $\mathrm{P}<0.05$ for the difference between $N$ $K B S N(l)$ and 2017 - winner (glove), and the difference between $N-K B S N(l)$ and 2019- winner( glove). The results indicate that the difference is statistically significant. We have dicsussed the current joint-embedding VQA models in detail in the Introduction secction and found that all existing models use static word vectors for text characterization ignoring the fact that the same word may represent different meanings in different contexts, and may also be used as different grammatical components. Taking into the problem brought by the static word vectors into account, our experiment constructs a joint embedding model based on dynamic word vector -- none KB specific network (N-KBSN) model and proves that it can improve the accuracy of the results through the comparison shown in the section4.1 and section4.3.

444 We simply propose the thought that the introduction of dynamic word vector into VQA system can improve the accuracy and do a simple experiment to find out the results, so the findings are preliminary and can be improved a lot in the future.For exemple, since the image feature characterization method is not the research content of this paper, we directly use the pre-trained Faster R-CNN network to extract image features. The design of parameters also migrates the champion model of VQA challenge in 2017, and only fine tune the parameters in the training. If we want to further improve the accuracy of the model, on the one hand, we can search the network to obtain the best combination of hyperparameters, on the other hand, we can use better image recognition models such as Mask R-CNN.

\section{3}

454

\section{6、Conclusion}

This paper constructs and tests a series of VQA models based on static word vector and dynamic word vector of different parameter configurations. The experimental results show that the $N$ $K B S N(l)$ proposed in our experiment has better performance than the other 2017 - winner (glove) and 2019 - winner (glove)models.Consequently, the introduction of dynamic word vector into the VQA model improves the accuracy of the results to some extent.

\section{Acknowledgement}

This work was jointly supported by the Sichuan Science and Technology Program (2019YJ0189) and the Fundamental Research Funds for the Central Universities (ZYGX2019J059) 


\section{Reference}

463

464

465

466

467

468

469

470

471

472

473

474

475

476

477

478

479

480

481

482

483

484

485

486

487

488

489

490

491

492

493

494

495

496

497

498

499

500
Anderson P, He X, Buehler C, Teney D, Johnson M, Gould S, and Zhang L. 2018. Bottom-up and top-down attention for image captioning and visual question answering. Proceedings of the IEEE conference on computer vision and pattern recognition. p 6077-6086.

Antol S, Agrawal A, Lu J, Mitchell M, Batra D, Lawrence Zitnick C, and Parikh D. 2015. Vqa: Visual question answering. Proceedings of the IEEE international conference on computer vision. p 2425-2433.

Atkinson T, Baier H, Copplestone T, Devlin S, and Swan JJIToG. 2019. The text-based adventure AI competition. 11:260-266.

Chen K, Wang J, Chen L-C, Gao H, Xu W, and Nevatia RJapa. 2015. Abc-cnn: An attention based convolutional neural network for visual question answering.

Felzenszwalb PF, Girshick RB, McAllester D, Ramanan DJItopa, and intelligence m. 2009. Object detection with discriminatively trained part-based models. 32:1627-1645.

Fukui A, Park DH, Yang D, Rohrbach A, Darrell T, and Rohrbach MJapa. 2016. Multimodal compact bilinear pooling for visual question answering and visual grounding.

Gao H, Mao J, Zhou J, Huang Z, Wang L, and Xu W. 2015. Are you talking to a machine? dataset and methods for multilingual image question. Advances in neural information processing systems. p 2296-2304.

Gelšvartas J, Simutis R, Maskeliūnas RJCi, and neuroscience. 2016. User adaptive text predictor for mentally disabled Huntington's patients. 2016.

Girshick R. 2015. Fast r-cnn. Proceedings of the IEEE international conference on computer vision. p 1440-1448.

Girshick R, Donahue J, Darrell T, and Malik J. 2014. Rich feature hierarchies for accurate object detection and semantic segmentation. Proceedings of the IEEE conference on computer vision and pattern recognition. $\mathrm{p}$ 580-587.

Goyal Y, Khot T, Summers-Stay D, Batra D, and Parikh D. 2017. Making the V in VQA matter: Elevating the role of image understanding in Visual Question Answering. Proceedings of the IEEE Conference on Computer Vision and Pattern Recognition. p 6904-6913.

He K, Gkioxari G, Dollár P, and Girshick R. 2017. Mask r-cnn. Proceedings of the IEEE international conference on computer vision. p 2961-2969.

He K, Zhang X, Ren S, Sun JJItopa, and intelligence m. 2015. Spatial pyramid pooling in deep convolutional networks for visual recognition. 37:1904-1916.

Ilievski I, Yan S, and Feng JJapa. 2016. A focused dynamic attention model for visual question answering.

Johnson J, Hariharan B, van der Maaten L, Fei-Fei L, Lawrence Zitnick C, and Girshick R. 2017. Clevr: A diagnostic dataset for compositional language and elementary visual reasoning. Proceedings of the IEEE Conference on Computer Vision and Pattern Recognition. p 2901-2910.

Klopfenstein LC, Delpriori S, and Ricci A. 2018. Adapting a Conversational Text Generator for Online Chatbot Messaging. International Conference on Internet Science: Springer. p 87-99.

Krishna R, Zhu Y, Groth O, Johnson J, Hata K, Kravitz J, Chen S, Kalantidis Y, Li L-J, and Shamma DAJIjocv. 2017. Visual genome: Connecting language and vision using crowdsourced dense image annotations. 123:32-73.

Ma L, Lu Z, and Li HJapa. 2015. Learning to answer questions from image using convolutional neural network. AAAI. Malinowski M, and Fritz M. 2014. A multi-world approach to question answering about real-world scenes based on

Peer] Comput. Sci. reviewing PDF | (CS-2020:08:52121:4:1:NEW 10 Dec 2020) 
501

502

503

504

505

506

507

508

509

510

511

512

513

514

515

516

517

518

519

520

521

522

523

524

525

526

527

528

529

530

531

532

533

534

535

536

537

538

539

540

541

uncertain input. Advances in neural information processing systems. p 1682-1690.

Malinowski M, Rohrbach M, and Fritz M. 2015. Ask your neurons: A neural-based approach to answering questions about images. Proceedings of the IEEE international conference on computer vision. p 1-9.

Noh H, Hongsuck Seo P, and Han B. 2016. Image question answering using convolutional neural network with dynamic parameter prediction. Proceedings of the IEEE conference on computer vision and pattern recognition. p 30-38.

Pennington J, Socher R, and Manning CD. 2014. Glove: Global vectors for word representation. Proceedings of the 2014 conference on empirical methods in natural language processing (EMNLP). p 1532-1543.

Peters ME, Neumann M, Iyyer M, Gardner M, Clark C, Lee K, and Zettlemoyer LJapa. 2018. Deep contextualized word representations. NAACL.

Redmon J, Divvala S, Girshick R, and Farhadi A. 2016. You only look once: Unified, real-time object detection. Proceedings of the IEEE conference on computer vision and pattern recognition. p 779-788.

Ren M, Kiros R, and Zemel R. 2015a. Exploring models and data for image question answering. Advances in neural information processing systems. p 2953-2961.

Ren S, He K, Girshick R, and Sun J. 2015b. Faster r-cnn: Towards real-time object detection with region proposal networks. Advances in neural information processing systems. p 91-99.

Russakovsky O, Deng J, Su H, Krause J, Satheesh S, Ma S, Huang Z, Karpathy A, Khosla A, and Bernstein MJjjocv. 2015. Imagenet large scale visual recognition challenge. 115:211-252.

Saito K, Shin A, Ushiku Y, and Harada T. 2017. Dualnet: Domain-invariant network for visual question answering. 2017 IEEE International Conference on Multimedia and Expo (ICME): IEEE. p 829-834.

Szegedy C, Liu W, Jia Y, Sermanet P, Reed S, Anguelov D, Erhan D, Vanhoucke V, and Rabinovich A. 2015. Going deeper with convolutions. Proceedings of the IEEE conference on computer vision and pattern recognition. p 1-9.

Tang Y, Liu S, Deng Y, Zhang Y, Yin L, Zheng WJCM, and Biomedicine Pi. 2020. Construction of force haptic reappearance system based on Geomagic Touch haptic device. 190:105344.

Vaswani A, Shazeer N, Parmar N, Uszkoreit J, Jones L, Gomez AN, Kaiser $Ł$, and Polosukhin I. 2017. Attention is all you need. Advances in neural information processing systems. p 5998-6008.

Wang P, Wu Q, Shen C, Dick A, Van Den Hengel AJItopa, and intelligence m. 2017. Fvqa: Fact-based visual question answering. 40:2413-2427.

Wang P, Wu Q, Shen C, Hengel Avd, and Dick AJapa. 2015a. Explicit knowledge-based reasoning for visual question answering.

Wang P, Wu Q, Shen C, van den Hengel A, and Dick AJae-p. 2015b. Explicit Knowledge-based Reasoning for Visual Question Answering. arXiv:1511.02570v2 arXiv. p arXiv:1511.02570.

Yin L, Li X, Zheng W, Yin Z, Song L, Ge L, and Zeng QJJoESS. 2019. Fractal dimension analysis for seismicity spatial and temporal distribution in the circum-Pacific seismic belt. 128:22.

Yu Z, Yu J, Cui Y, Tao D, and Tian Q. 2019. Deep modular co-attention networks for visual question answering. Proceedings of the IEEE conference on computer vision and pattern recognition. $\mathrm{p}$ 6281-6290.

Zheng W, Li X, Yin L, and Wang YJRL. 2016. Spatiotemporal heterogeneity of urban air pollution in China based on spatial analysis. 27:351-356.

Zhou B, Tian Y, Sukhbaatar S, Szlam A, and Fergus RJapa. 2015. Simple baseline for visual question answering.

Peer) Comput. Sci. reviewing PDF | (CS-2020:08:52121:4:1:NEW 10 Dec 2020) 
Figure 1

\section{N-KBSN infrastructure}

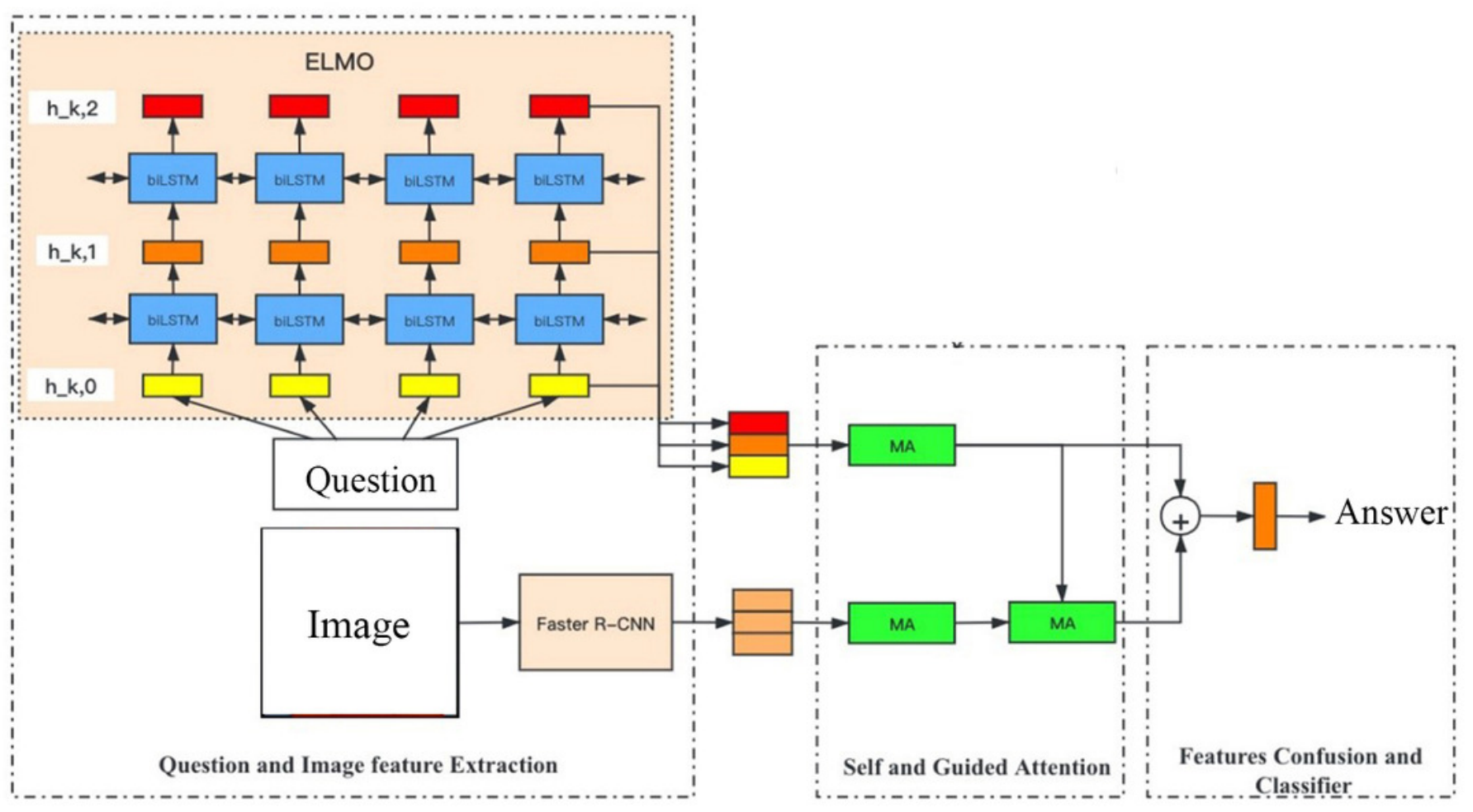


Figure 2

\section{UML.jpg}

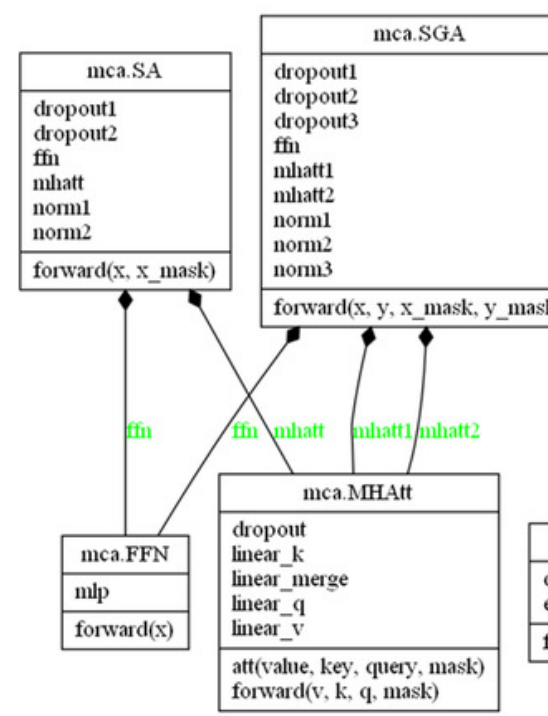

\begin{tabular}{|c|c|}
\hline mca.MCA_ED & net.AttFlat \\
\hline $\begin{array}{l}\text { dec_list } \\
\text { enc_list }\end{array}$ & $\begin{array}{l}\text { linear_merge } \\
\text { mlp }\end{array}$ \\
\hline forward( $\mathrm{x}, \mathrm{y}, \mathrm{x}$ mask, $\mathrm{y}$ _mask $)$ & forward $(\mathrm{x}, \mathrm{x}$ mask $)$ \\
\hline
\end{tabular}

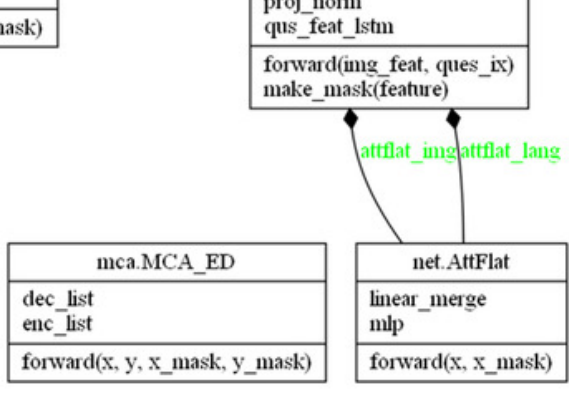

forward(v, k, q, mask)

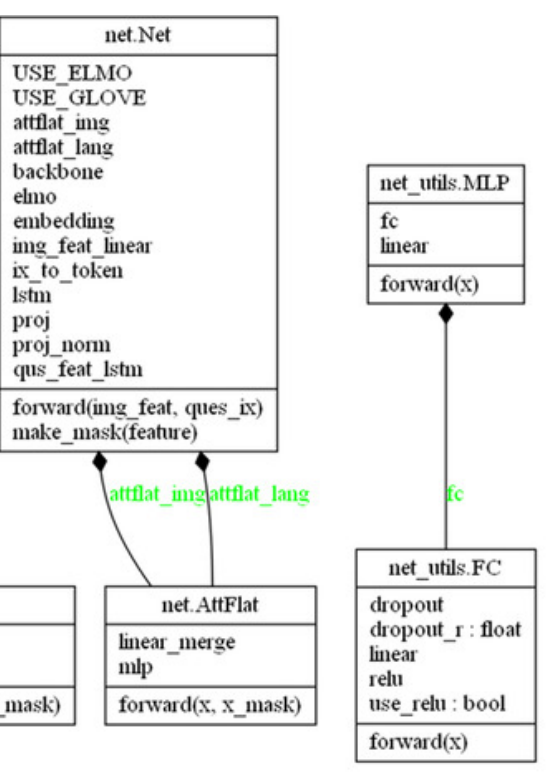

\begin{tabular}{|l|}
\hline net_utils.LayerNorm \\
\hline a_2 \\
b_2 \\
eps : float \\
\hline forward(x) \\
\hline
\end{tabular}

\begin{tabular}{|l|}
\hline optim.WarmupOptimizer \\
\hline batch_size \\
data_size \\
Ir_base \\
optimizer \\
\hline rate(step) \\
step0 \\
zero_grad0 \\
\hline
\end{tabular}


Figure 3

statistics chart of experimental results of $N-K B S N(m)$ and baseline (glove) in different training subsets

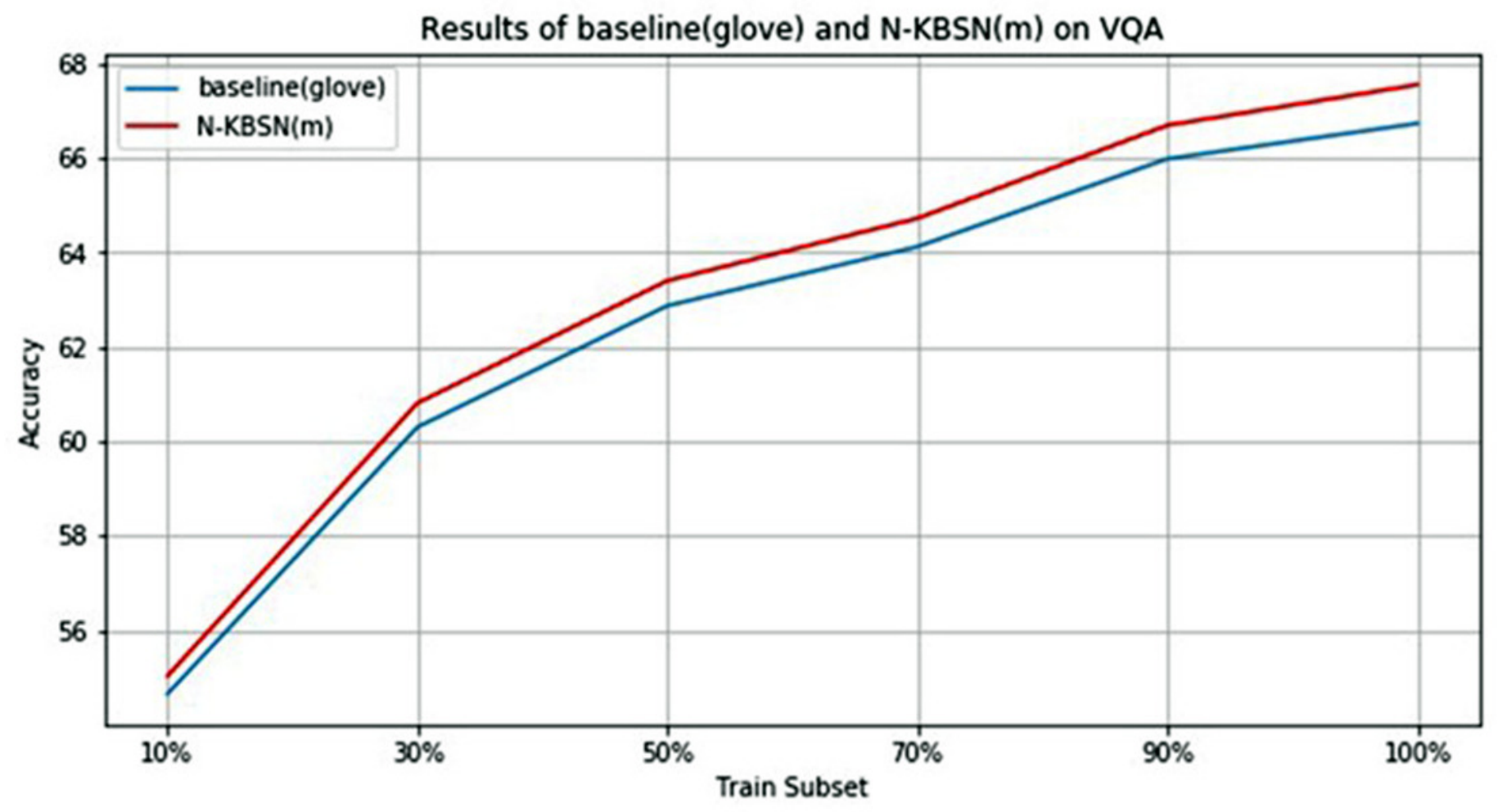


Figure 4

Sample_examples_of_validation_sets

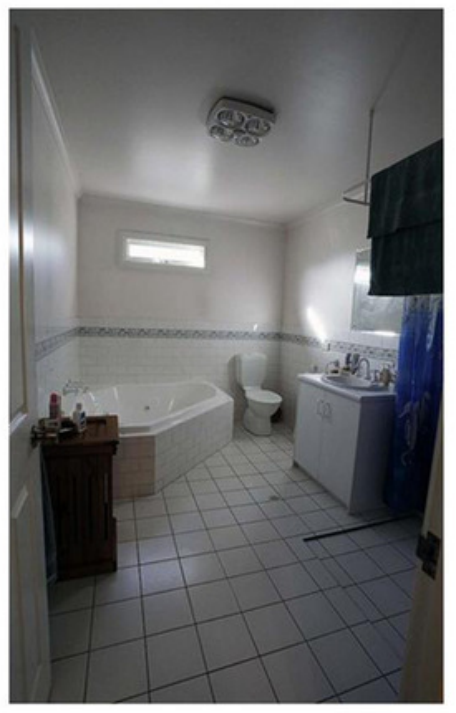

Q: What color is the shower curtain?

Res(elmo):

Res(glove):

\section{Blue}

White

(a)

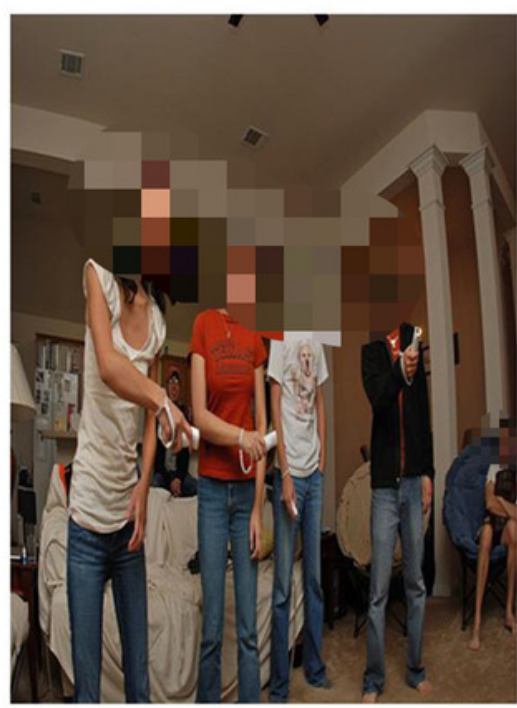

How many elderly people are in this picture?
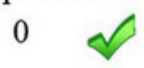

5

(b)

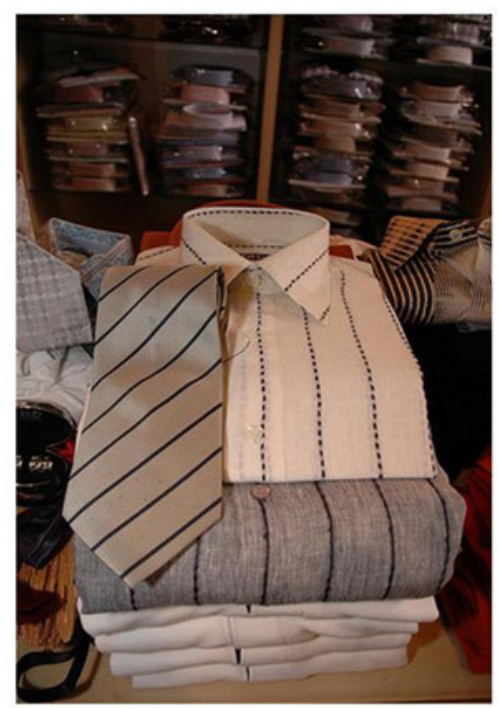

What fashion accessory is sitting on top of the white collared shirt? Tie

Hat

(c) 
Figure 5

Statistical results of difference between $\mathrm{N}-\mathrm{KBSN}(\mathrm{m})$ model and baseline (glove) model

Test Statistics ${ }^{a}$

\begin{tabular}{lr} 
& $\begin{array}{c}\text { difference - } \\
\text { NKBSN_M }\end{array}$ \\
\hline$Z$ & $-2.201^{\mathrm{b}}$ \\
\hline Asymp. Sig. (2-tailed) & .028 \\
\hline
\end{tabular}

a. Wilcoxon Signed Ranks Test

b. Based on positive ranks.

(A)

\section{Report}

\begin{tabular}{c|r|r}
$\begin{array}{c}\text { Mean } \\
\text { baselineGlov } \\
e\end{array}$ & NKBSN_M & difference \\
\hline 62.4417 & 63.0333 & .5917 \\
\hline
\end{tabular}


Figure 6

Sample examples of validation sets

\section{Test Statistics ${ }^{a}$}

\begin{tabular}{lr} 
& $\begin{array}{c}\text { difference - } \\
\text { winnerGlove2 } \\
017\end{array}$ \\
\hline $\mathrm{Z}$ & $-2.023^{\mathrm{b}}$ \\
\hline Asymp. Sig. (2-tailed) & .043 \\
\hline
\end{tabular}

a. Wilcoxon Signed Ranks Test

b. Based on positive ranks.

(A)

\section{Report}

Mean

\begin{tabular}{c|c|r} 
NKBSNL & $\begin{array}{c}\text { winnerGlove2 } \\
017\end{array}$ & difference \\
\hline 68.8900 & 62.6140 & 6.2760 \\
\hline
\end{tabular}

(B) 
Figure 7

Statistical results of difference between N-KBSN(I) model and 2019winner (glove) model

Test Statistics ${ }^{\mathrm{a}}$

\begin{tabular}{lr} 
& $\begin{array}{c}\text { difference - } \\
\text { winnerGlove2 } \\
019\end{array}$ \\
\hline Z & $-2.023^{\mathrm{b}}$ \\
\hline Asymp. Sig. (2-tailed) & .043 \\
\hline
\end{tabular}

a. Wilcoxon Signed Ranks Test

b. Based on positive ranks.

(A)

\section{Report}

\begin{tabular}{|c|c|c|}
\hline NKBSNL & $\begin{array}{c}\text { winnerGlove2 } \\
019\end{array}$ & difference \\
\hline 68.8900 & 68.4660 & .4240 \\
\hline
\end{tabular}




\section{Table $\mathbf{1}$ (on next page)}

comparison of representative joint embedding models 
Table 1 comparison of representative joint embedding models

2

\begin{tabular}{|c|c|c|c|c|c|}
\hline Model & $\begin{array}{c}\text { Image } \\
\text { characterization }\end{array}$ & $\begin{array}{c}\text { Text } \\
\text { characterization }\end{array}$ & Feature fusion & $\begin{array}{c}\text { VQA } \\
\text { accuracy(\%) }\end{array}$ & $\begin{array}{l}\text { Static word } \\
\text { vector }\end{array}$ \\
\hline LSTMQ+I & VGGnet & LSTM & $\begin{array}{c}\text { Itemized } \\
\text { multiplication }\end{array}$ & 54.1 & Yes \\
\hline iBOWIMG & GoogleNet & BoW & series connection & 55.9 & Yes \\
\hline DPPNet & VGGnet & GRU & $\begin{array}{c}\text { Dynamic parameter } \\
\text { layer }\end{array}$ & 57.4 & Yes \\
\hline $\mathrm{D}-\mathrm{CNN}$ & $\mathrm{CNN}$ & $\mathrm{CNN}$ & $\mathrm{CNN}$ & 58.4 & Yes \\
\hline $\mathrm{MCB}$ & RestNet & LSTM & $\mathrm{MCB}$ & 64.2 & Yes \\
\hline 2017-winer & Faster R-CNN & Glove+GRU & $\begin{array}{c}\text { Itemized } \\
\text { multiplication }\end{array}$ & 69.87 & Yes \\
\hline 2018-winer & Faster R-CNN & Glove+GRU & $\begin{array}{c}\text { Itemized } \\
\text { multiplication }\end{array}$ & 72.27 & Yes \\
\hline 2019-winer & Faster R-CNN & Glove+LSTM & MLP & 75.26 & Yes \\
\hline
\end{tabular}




\section{Table 2 (on next page)}

Parameter configuration of ELMO 
Table 2 Parameter configuration of ELMo

\begin{tabular}{ccccc}
\hline Model & Parameters (M) & LSTM Size & Output Size & ELMo Size \\
\hline$E L M o_{s}$ & 13.6 & 1024 & 128 & 256 \\
$E L M o_{m}$ & 28.0 & 2048 & 256 & 512 \\
$E L M o_{l}$ & 93.6 & 4096 & 512 & 1024 \\
\hline
\end{tabular}

2 


\section{Table 3 (on next page)}

Statistics information of word2vec, glove and three Elmo models 
1

2

\begin{tabular}{lccc}
\multicolumn{5}{c}{ Table 3 Statistical information of word2vec, glove, three ELMo models } \\
\hline name & Pre-training corpus (size) & $\begin{array}{c}\text { Word vector } \\
\text { dimension }\end{array}$ & $\begin{array}{c}\text { Number of } \\
\text { word vectors }\end{array}$ \\
\hline $\begin{array}{l}\text { word2vec } \\
\text { Glove }\end{array}$ & Wikipedia 2014 + Gigaword 5 (Six billion words) & 300 & 400 thousand \\
& & 300 & \\
\hline$E L M o_{s}$ & WMT 2011 (800 million words) & 256 & \\
$E L M o_{m}$ & 512 & \\
$E L M o_{l}$ & & 1024 & \\
\hline
\end{tabular}




\section{Table 4 (on next page)}

The results of different text features characterization model in the Val dataset 
Table 4 The results of different text features characterization model in the Val dataset

\begin{tabular}{lllll}
\hline Model & Accuracy & Yes or No & Count & Others \\
\hline baseline (random) & 62.34 & 78.77 & 41.92 & 55.27 \\
2017-winner(glove) & 63.22 & 80.07 & 42.87 & 55.81 \\
baseline (w2v) & 64.37 & 81.89 & 44.51 & 56.31 \\
baseline(glove) & 66.73 & 84.56 & 49.52 & 57.72 \\
2019-winner(glove) & 67.22 & 84.80 & 49.30 & 58.60 \\
\hline$N-K B S N(s)$ & 67.27 & 84.76 & 49.31 & 58.73 \\
$N-K B S N(m)$ & 67.55 & 85.03 & 49.62 & 59.01 \\
$N-K B S N(l)$ & $\mathbf{6 7 . 7 2}$ & $\mathbf{8 5 . 2 2}$ & $\mathbf{4 9 . 6 3}$ & $\mathbf{5 9 . 2 0}$ \\
\hline
\end{tabular}

2 


\section{Table 5 (on next page)}

statistics of experimental results of $N-\operatorname{KBSN}(m)$ and baseline (glove) in different training subsets 
1 Table5 statistics of experimental results of $N-K B S N(m)$ and baseline (glove) in different training subsets

\begin{tabular}{llll}
\hline Accuracy & baseline (glove) & $N-K B S N(m)$ & Difference \\
\hline training subset(10\%) & 54.65 & $\mathbf{5 5 . 0 3}$ & 0.38 \\
training subset(30\%) & 60.30 & $\mathbf{6 0 . 8 1}$ & 0.51 \\
training subset(50\%) & 62.87 & $\mathbf{6 3 . 4 0}$ & 0.53 \\
training subset(70\%) & 64.12 & $\mathbf{6 4 . 7 2}$ & 0.60 \\
training subset(90\%) & 65.98 & $\mathbf{6 6 . 6 9}$ & 0.71 \\
training subset(100\%) & 66.73 & $\mathbf{6 7 . 5 5}$ & 0.82 \\
\hline
\end{tabular}

2 


\section{Table 6(on next page)}

The difference between the answers of Res(elmo)and Res (glove) in the validation set 
Table 6 The difference between the answers of Res(ElMo)and Res (glove) in the val set

The number of questions with different answers (proportion) 52,990(24.7\%)

Only Res(ElMo) answered correctly(proportion)14,711(27.8\%)

Only Res(glove) answered correctly(proportion)13,991 (26.4\%)

Both answered wrongly (proportion) $24288(45.8 \%)$

Both answered correctly (proportion) $0(0 \%)$

2 


\section{Table 7 (on next page)}

Comparison of the results of $N-K B S N(I)$ model and other models on test set 
Table 7Comparison of the results of $N-K B S N(l)$ model and other models on test set

\begin{tabular}{|c|c|c|c|c|c|}
\hline \multirow{2}{*}{ Model } & \multicolumn{4}{|c|}{ Test-dev } & \multirow{2}{*}{$\begin{array}{l}\text { Test-std } \\
\text { All }\end{array}$} \\
\hline & All & $\mathrm{Y} / \mathrm{N}$ & Num & Other & \\
\hline 2017 — winner (glove) & 65.32 & 81.82 & 44.21 & 56.05 & 65.67 \\
\hline 2019 - winner (glove) & 70.63 & 86.82 & 53.26 & 60.72 & 70.90 \\
\hline$N-K B S N(l)$ & 71.14 & 87.13 & 54.05 & 60.90 & 71.23 \\
\hline
\end{tabular}

